\title{
The Transcervical Extended Access, a Feasible Approach for the Surgical Treatment of Benign Tumors of the Posterior Mediastinum?
}

\author{
David Perez, Francisco Hernandez, Jose Ramon Cano, Wolker Tavarez, Gara Torrent, \\ Santiago Quevedo, Luis Lopez \\ Department of Thoracic Surgery, Insular Materno-Infantil University Hospital, Las Palmas de Gran Canaria, Spain \\ Email: dperalo@live.com
}

How to cite this paper: Perez, D., Hernandez, F., Cano, J.R., Tavarez, W., Torrent, G., Quevedo, S. and Lopez, L. (2018) The Transcervical Extended Access, a Feasible Approach for the Surgical Treatment of Benign Tumors of the Posterior Mediastinum? Surgical Science, 9, 128-133. https://doi.org/10.4236/ss.2018.93014

Received: January 15, 2018

Accepted: March 25, 2018

Published: March 28, 2018

Copyright $\odot 2018$ by authors and Scientific Research Publishing Inc. This work is licensed under the Creative Commons Attribution International License (CC BY 4.0).

http://creativecommons.org/licenses/by/4.0/

\begin{abstract}
Transcervical approach for tumors of the posterior mediastinum is traditionally thought not to be indicated. Hereby we report on a case of a patient with a huge neurogenic tumor of the posterior mediastinum which was successfully excised through a transcervical extended approach and, additionally, the variety of surgical approaches used to remove tumors of the posterior mediastinum is discussed. The new refined techniques of transcervical extended mediastinal operations, which are recently gaining popularity among surgeons, allowed for a safe dissection of the tumor, thus patient could benefit from a short painless postoperative course. The authors suggest that surgeons trained in these particular techniques should consider the choice of the transcervical extended access in selected cases of benign tumors of the posterior mediastinum.
\end{abstract}

\section{Keywords}

Mediastinum, Neurogenic Neoplasms, Thoracic Surgery, Transcervical Access

\section{Introduction}

Tumors in the posterior mediastinum are uncommon. Among these, Schwannomas are benign, slow-growing neoplasms that originate from the proliferation of Schwann cells of the sheath nerve of the spinal root or intercostals nerve cells contained within a capsule. They typically occur in asymptomatic young adults or adolescents [1], but when they achieve a large size, these tumors cause symptoms due to direct effects of the mediastinal mass, as chest discomfort, dyspnea or dysphagia, the two latter ones being consequences of tracheal or esophageal 
compression, respectively. Grossly, thoracic tumors are habitually fusiform or spherical and capsulated, but when they attain large sizes growing into the spinal canal and paravertebral region, they acquire a characteristic dumbbell shape [2]. Once imaging suggests the benign neurogenic origin of the tumor, surgery is required for definitive histological diagnosis and for treatment of symptoms and signs. Due to the complex anatomy of the mediastinum, preoperative high-resolution imaging techniques, computed tomography (CT) and magnetic resonance imaging (MRI), have to be carried out in order to accurately assess the morphology of the tumor and its exact anatomical location [3]. The correct operative approach to localized mediastinal neurogenic tumors should be determined by the surgical team based on the information provided by preoperative imaging and on the preference and experience of the surgeon performing the resection. A number of different approaches for the resection of cervicothoracic paravertebral neoplasms have been described, each providing differing visualization of the anatomy and having specific limitations and morbidity. We hereby report on a case of a large Schwannoma of the posterior mediastinum resected by the new transcervical extended access; the variety of surgical approaches used to remove tumors of the posterior mediastinum is additionally discussed.

\section{Case Report}

A 21-year-old non-smoker male patient with no previous history of clinical disease was referred to our surgical department by his general practitioner under suspicion of malignant intrathoracic mass. Patient reported a two-month history of non-specific chest discomfort and dry cough. Routine blood investigations carried out at the family physicians clinic were within normal limits, but chest $\mathrm{X}$-ray revealed a mass in the left paravertebral gutter creating obtuse angles with the lung. At our surgical department high-resolution imaging techniques were performed. Contrast enhanced CT scan showed a well-circumscribed mass of 6.5 $\mathrm{cm}$ at its largest area, from D2 to D4 vertebral levels, in the left paravertebral region, while MRI reported intermediate-signal intensity on T1-weighted images, all suggesting primary mediastinal tumor of neurogenic origin. Patient was directly taken to the operation room for complete excision of the mass since preoperative confirmatory fine-needle aspiration cytology or biopsy were considered not to be helpful in a suspected case of benign neurogenic tumor. With the patient positioned in a supine position under general anesthesia with single lumen endotracheal intubation, the transcervical extended procedure was performed following the surgical steps as they were described by Marcin Zielinski, the original author of this technique [4]. Procedure included a $8 \mathrm{~cm}$ collar incision in the neck, the carefully dissection of the arterial and venous trunks of the supra-aortic area, the elevation of the sternal manubrium with a hook and bilateral visualization of the laryngeal recurrent and vagus nerves, all in an open manner. In this case, assistance with the mediastinoscopy was not found necessary. Freed of the capsulated tumor by finger blunt dissection and with cautery, applied only 
at its adhesions, allowed its complete extraction without operative complications. Drains were not left since mediastinal pleura was not violated. The postoperative period was uneventful and patient was discharged from hospital at the second day after surgery with no complaints. Histopathological examination of the mass confirmed schwannoma.

\section{Discussion}

Primary treatment modality for benign neurogenic tumors of the mediastinum is en-bloc surgical resection. Before video-assisted thoracic surgery (VATS) was used, the preferred surgical procedure for resection of posterior mediastinal benign masses was the high posterolateral thoracotomy [5]. VATS is actually considered the surgical approach of choice for diagnosing and resecting tumors smaller than $7 \mathrm{~cm}$ without signs of malignancy since it has better outcomes in terms of surgical procedure, amount of blood loss, length of drainage time, and length of hospital stay [6] [7]. Midline approaches through sternotomy or transmanubrial approach are alternatives that secure an adequate visual field and enable the safe separation of blood vessels and nerves from the tumor [8]; however, anterior approaches will generally not provide adequate exposure for masses located deep in the posterior mediastinum or that extend below the level of the pulmonary hilum [9].

With regard to the standard transcervical operative access, although enabling a suitable exposure and access to perform the operation for most of the benign pathology and favourable malignancies of the superior or medial mediastinum, it is not considered appropriate for the resection of masses located at the posterior or low mediastinum because of the depth of the required dissection and the potential risk of damaging vessels and nerves in the upper mediastinum. In this paper we report on the successful surgical resection of a huge Schwannoma of the posterior mediastinum through the more refined recently described techniques of extended transcervical access [10]. In the reported case, despite the big size and posterior location of the mass (see Figure 1), the technique of transcervical extended mediastinal dissection (see Figure 2) provided a safe separation of the tumor from vessels and nerves avoiding thoracothomy. In absence of big incisions or postoperative drains-pleural spaces were not violated, the patient could benefit from a short painless uneventful postoperative course.

The new used route of access to the mediastinum briefly described in this paper, called TEMLA, was developed by Marcin Zielinski in 2004 as a technique for staging of the mediastinal lymph nodes in patients with non-small cell lung cancer [10]. The TEMLA technique is performed through a 5- to 8-cm collar incision in the neck and enables the surgical access to all mediastinal compartments for removal of the mediastinal nodes in semiopen fashion. This technique includes dissection of the supra-aortic trunks, visualization of the laryngeal recurrent and vagus nerves and ligature and section of the right and left inferior thyroideal pedicles. The elevation of the sternum by the use of a hook inserted 


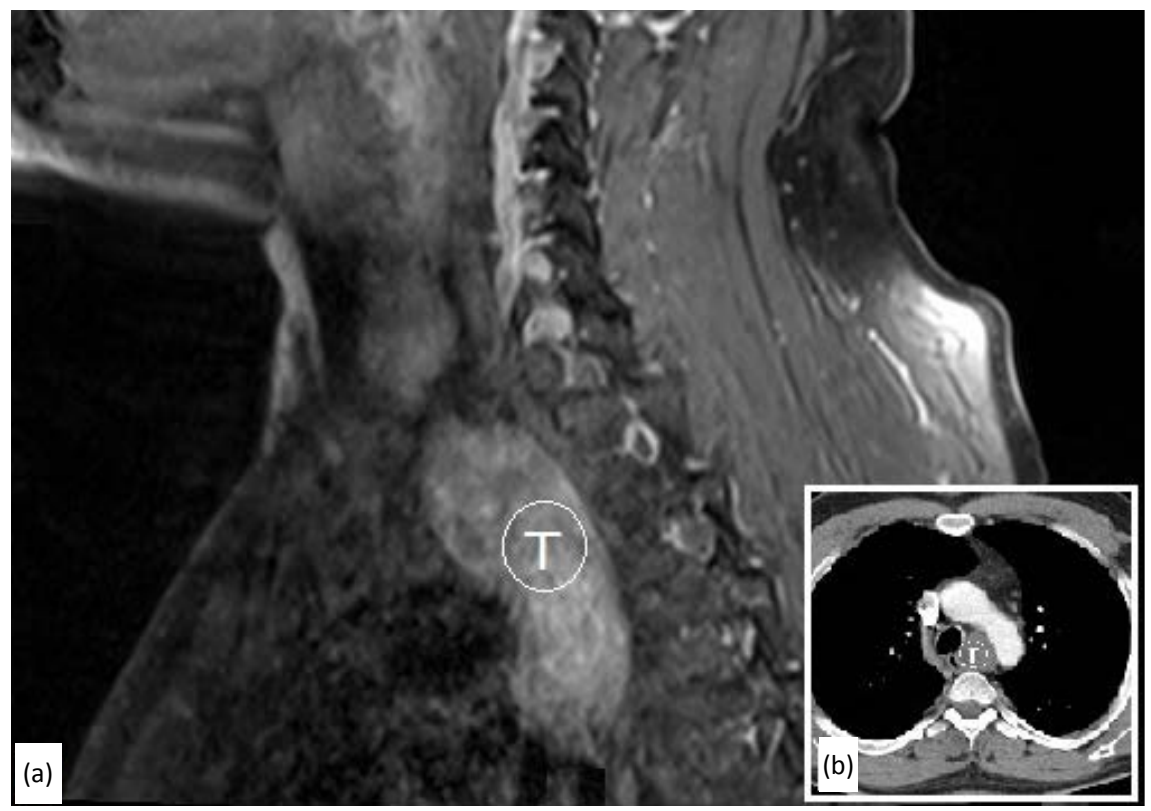

Figure 1. Sagittal magnetic resonance image (a) and axial view of the CT scan (b) showing a $6.5 \mathrm{~cm}$ well-defined mass $(\mathrm{T})$ located in the posterior mediastinum.

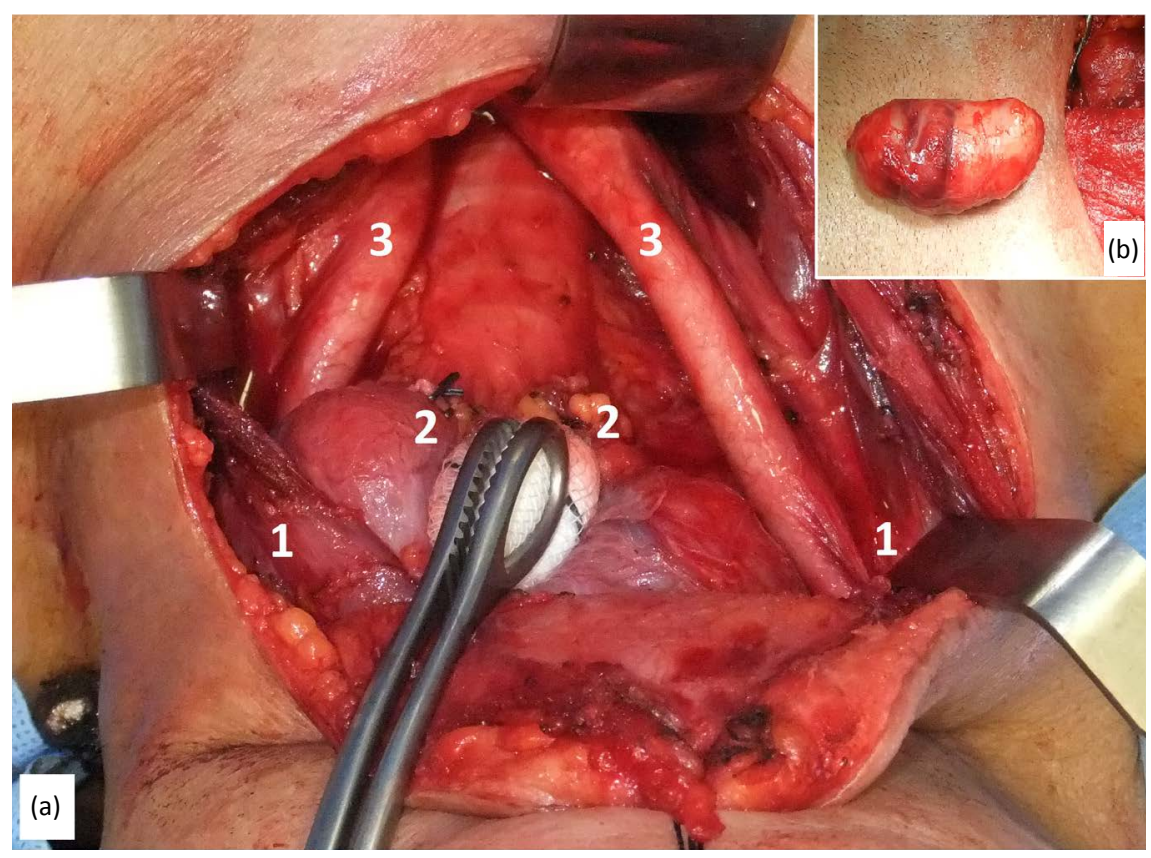

Figure 2. (a) Operative images showing some of the operative steps of the extended transcervical access which enable the access to the mediastinum: the lateral retraction of sternocleidmastoideus and extrinsic laringealx muscles (1), the section of the lower pedicles of the thyroid gland (2) allowing the medial mobilization of the cervicovisceral axis, and the opening of the sheath of the common carotid arteries (3). (b) Operative image of the excised neurogenic tumor.

under the manubrium combined with the lateral mobilization of the cervicovisceral axis (i.e., larynx trachea esophagus) allow for a broad access to the anterior, medial and posterior mediastinum. In Zielinski's experience, the extended tran- 
scervical approach is also very valuable for the resection of tumors of the superior or medial mediastinum, thymectomy, closure of the bronchial stump postpneumonectomy fistula and pulmonary resections; only tumors located in the inferior mediastinum could not be removed with this technique.

However, although since it was published, the transcervical extended procedure was well received by the international scientific community. Few other authors have published their experiences with this operative access [11].

\section{Conclusion}

Authors believe that, when comparing with thoracothomy or VATS, transcervical extended procedure provides benefits for patients with mediastinal tumors, since it offers a less painful postoperative course and shorter hospital stay. For these reasons, we suggest surgeons trained in these particular techniques to consider the choice of the transcervical extended access for the operative approach of selected well-encapsulated masses from the posterior mediastinum.

\section{References}

[1] Strollo, D.C., Rosado-de-Christenson, M.L. and Jett, J.R. (1997) Primary Mediastinal Tumors: Part II. Tumors of the Middle and Posterior Mediastinum. Chest, 112, 1344-1357. https://doi.org/10.1378/chest.112.5.1344

[2] Nakazono, T., White, C.S., Yamasaki, F., Yamaguchi, K., Egashira, R., Irie, H. and Kud, S. (2011) MRI Findings of Mediastinal Neurogenic Tumors. American Journal of Roentgenology, 197, W643-W652. https://doi.org/10.2214/AJR.10.6119

[3] Cardillo, G., Carleo, F., Khalil, M.W., Carbone, L., Treggiari, S., Salvadori, L., Petrella, L. and Martelli, M. (2008) Surgical Treatment of Benign Neurogenic Tumours of the Mediastinum: A Single Institution Report. European Journal of Cardio-Thoracic Surgery, 34, 1210-1214. https://doi.org/10.1016/j.ejcts.2008.09.006

[4] Zieliński, M. (2014) Transcervical Resection of Mediastinal Tumors. In: Zieliński, M. and Rami-Porta, R., Eds., The Transcervical Approach in Thoracic Surgery, Springer, New York, 141-147. https://doi.org/10.1007/978-3-642-54565-8_14

[5] Topçu, S., Alper, A., Gülhan, E., et al. (2000) Neurogenic Tumours of the Mediastinum: A Report of 60 Cases. Canadian Respiratory Journal, 7, 261-265. https://doi.org/10.1155/2000/782325

[6] Zhang, Z., Liu, D., Guo, Y., Shi, B., Tian, Y., Song, Z. and Liang, C. (2012) Preferred Surgical Procedure for Posterior Mediastinal Neurogenic Tumor. Thoracic Cancer, 3, 68-71. https://doi.org/10.1111/j.1759-7714.2011.00074.x

[7] Riquet, M., Mouroux, J., Pons, F., et al. (1995) Videothoracoscopic Excision of Thoracic Neurogenic Tumors. The Annals of Thoracic Surgery, 60, 943-946. https://doi.org/10.1016/0003-4975(95)00487-6

[8] Li, W.W.L., van Boven, W.J.P., Annema, J.T., Eberl, S., Klomp, H.M. and de Mol, B.A.J.M. (2016) Management of Large Mediastinal Masses: Surgical and Anesthesiological Considerations. Journal of Thoracic Disease, 8, E175-E184. https://doi.org/10.21037/jtd.2016.02.55

[9] Cherqui, A., Kim, D.H., Kim, S.H., Park, H.K. and Kline, D.G. (2007) Surgical Approaches to Paraspinal Nerve Sheath Tumors. Neurosurgical Focus, 15, E9.

[10] Zieliński, M., Czajkowski, W., Kosinski, S., Fryzlewicz, E., Nabialek, T., Pankowski, 
J., et al. (2017) Transcervical Approach for Thoracic Operations. Clinics in Surgery, 2, 1447.

[11] Pérez-Alonso, D., Cano-García, J.R., Quevedo-Losada, S. and López-Rivero, L. (2010) TEMLA: A New Technique for the Treatment of Recurrent Thyroid Cancer. Cirugía Española, 88, 427-429. 